(C) 1982. The Genetical Society of Great Britain

\title{
ALLOZYME VARIATION IN THE INBREEDING SPECIES LOLIUM TEMULENTUM L.
}

\author{
M. D. HAYWARD, and M. T. M. ZARUK* \\ Welsh Plant Breeding Station, Aberystwyth, Dyfed, SY23 3EB, U.K.
}

Received 17.v.82

\section{INTRODUCTION}

EARLIER studies of the autogamous species Lolium temulentum revealed considerable variability for quantitative characters within several populations (Hayward and Jackson, 1971). Individual plants within each collection, which originated from botanical gardens, were found to segregate for one or more quantitative characters when selfed progeny were examined. This variation was accounted for by mechanisms maintaining heterozygosity or by occasional outcrossing having occurred in this supposedly self-pollinating species.

The present experiment examines the enzyme phenotypes of two wild populations of Lolium temulentum and briefly relates these results to aspects of population structure, the breeding system and the biology of this species.

\section{MATERIALS AND METHODS}

Lolium temulentum $\mathrm{L}$. is of widespread occurrence and has long been recognised as a weed of cereal crops in the Mediterranean regions of Europe and Asia (Terrell, 1968). It is an annual self-fertilizing species even though occasional open florets with exserted anthers can be found (Beddows, 1931). Two populations were obtained by plant collecting expeditions of the Welsh Plant Breeding Station.

Population 1 consisted of inflorescences from 22 individual plants growing amongst a crop of Triticum aestivum on peaty soil, west of Lake Cildu, Turkey. Seed from these single inflorescences, maintained as distinct lines was used to provide five seedlings of each family for electrophoretic examination.

Population 2 derived from a collection of 27 single inflorescences again from amongst a wheat crop growing near Aral, Iran. For this population, seed of the original mother plants was no longer available but the selfed progeny of five plants deriving from each of the mother plants was utilized. Single plants were established of each progeny lot to form families of five plants.

\section{(i) Isozyme assay}

Horizontal starch gel electrophoresis using modifications (Hayward and McAdam, 1977) of the method of Scandalios (1969), was employed for the separation of enzyme variants. The systems examined were for the

* Present Address: Central Agricultural Research Institute, Dept. of Agriculture, Perandenya, Sri Lanka. 
enzymes acid phosphatase (ACP), phosphoglucoisomerase (PGI) and glutamate-oxaloacetate-transaminase (GOT), which are coded for by one, two and three loci respectively in other closely related Lolium species (Hayward and McAdam, 1977). The nomenclature of the differing isozymes refers to standard migration distances of control extracts of Lolium perenne, "a" being the most anodal band.

\section{REsults AND Discussion}

The numbers of families in the two populations with the differing isozyme phenotypes discovered are presented in table 1 . The range of isozymes is comparable to other Lolium species. Genetic analyses have confirmed that these are also under the control of the same number of loci, with co-dominant alleles, as in other members of the genus (Hayward and McAdam, 1977; Zaruk, 1979). Direct observation of genotype and allele frequencies can thus be made without recourse to breeding tests.

TABLE 1

Numbers of families of each phenotype in the two populations

\begin{tabular}{lccc} 
& & \multicolumn{2}{c}{ Population } \\
Locus & Phenotype & Turkey & Iran \\
Pgi-2 & $a a$ & 6 & 27 \\
& $c c$ & 16 & - \\
Got-1 & $a a$ & 6 & 27 \\
& $b b$ & 16 & - \\
Got-2 & $a a$ & 22 & 27 \\
Got-3 & $c c$ & 22 & 27 \\
Acp-1 & $b b$ & 21 & 27 \\
& $c c$ & 1 & -
\end{tabular}

These earlier surveys of a large number of populations and cultivars of $L$. perenne and $L$. multiflorum had shown the Got-1 locus to be monomorphic. However, in L. temulentum at least two alleles are present. As in the other Lolium species the Pgi-1 allozymes cannot be clearly distinguished under the electrophoretic conditions employed (Hayward and McAdam, 1977).

The results shown in table 1 reveal two contrasting patterns of population structure. The Iranian population is completely monomorphic at each locus whereas the Turkish population is more typical of many inbreeding species in that variation occurs at three of the loci; this variation is however in the homozygous state. The genetic differentiation of these two populations is clearly shown in a comparison of the different alleles found within each. In the polymorphic Turkish population there is a predominance of one allele at each locus, which occurs with a frequency of up to 96 per cent for $A c p-1 b$. In the monomorphic Iranian population it is the rarer alleles of the polymorphic loci in the Turkish population which are fixed at two out of the three variable loci. It is of interest to note in this latter population that no evidence of heterozygosity for isozyme loci was found indicating that inbreeding must be complete and that no selective mechanisms are operative in maintaining heterozygosity as is often encountered in inbreeding species (Brown, 1979). The limited number of loci examined 
here may of course give a false impression of population structure, since analyses of quantitative characters, in two generations of a selfing series in both populations, indicated the existence of variation in a number of morphological and physiological characters (Zaruk, 1979). This took the form of heterogeneity between families but only in the Turkish population was this associated with further segregation, suggesting the presence of heterozygosity. Undoubtedly, these quantitative characters are of greater adaptive significance and hence likely to be subjected to mechanisms which maintain potential variability.

The occurrence of adaptive gene complexes in conserving variation is further revealed by consideration of possible multi-locus associations. Although the number of individuals examined from the polymorphic Turkish collection is low, associations were found between the Pgi-2 locus and $G o t-1$ where all $6 a a$ phenotypes at the former locus are also $a a$ at the second locus and similarly for the $16 b b$ phenotypes at Pgi-2 which are all $b b$ at Got-1. This distribution is clearly heterogenous $\left(\chi_{[2]}^{2}=17 \cdot 2\right.$, $P=0.001$ ). These "linkages" are not fixed because recombinants have been found in a limited number of crossed progeny examined. The associations in the original population would suggest the possibility of adaptive epistatic combinations of genes maintained by some form of balancing type of selection. Such a selective process will aid the development and maintenance of polymorphisms at both loci (Allard and Kahler, 1971).

The patterns of variation exhibited by these two populations may be accounted for by Lolium temulentum being an obligate weed of cereal crops (Terrell, 1968). In association with cereal cropping systems it must regularly pass through severe bottlenecks which may considerably restrict the variability found, as in the present case for the Iranian population. Although these selective forces may be severe, variability can still be encountered within some populations providing the potential for future evolutionary change.

Acknowledgements.-M. T. M. Zaruk acknowledges the financial support of the British Council which allowed him to pursue the MSc course in plant breeding at the University of Wales. We wish to thank N. J. McAdam for expert technical assistance.

\section{REFERENCES}

ALLARD, R. W., AND KAHLER, A. L. 1971. Allozyme polymorphisms in plant populations. Stadler Symposia, (University of Missouri) 3, 9-24.

BEDDOWS, A. R. 1931. Seed setting and flowering in various grasses. In: Self- and CrossFertility and Flowering Habits of Certain Herbage Grasses and Legumes. Bulletin Series H, No. 12, p. 70, Welsh Plant Breeding Station. Aberystwyth.

BROWN, A. H. D. 1979. Enzyme polymorphisms in plant populations. Theor. Pop. Biol., 15, $1-42$.

HAYWARD, M. D., AND JACKSON, J. C. 1971. The genetic organisation of populations of the inbreeding species Lolium temulentum. Heredity, 26, 323-326.

HAYWARD, M. D., AND MCADAM, N. J. 1977. Isozyme polymorphisms as a measure of distinctness and stability in cultivars of Lolium perenne. Z. Pflanzenzuchtg., 79, 59-68.

SCANDALIOS, J. C. 1969. Genetic control of multiple molecular forms of enzymes in plants-a review. Biochem. Gen., 3, 37-79.

TERRELL, E. F. 1968. A taxonomic revision of the genus Lolium. USDA Tech. Bull. 1392. ZARUK, M. T. M. 1979. Genetic variation and the breeding system in Lolium species. M.Sc. Thesis, University Coll., Wales, Aberystwyth. 